\title{
SUMS OF DISTINCT DIVISORS AND SUMS OF DISTINCT UNITS
}

BERNARD JACOBSON

1. Introduction. For a given positive integer $M$, let $\alpha(M)$ be the number of integers $n$ which can be written in the form

$$
n=\sum d
$$

where the $d$ are distinct positive divisors of $M$. Clearly $\alpha(M) \leqq \sigma(M)$. In a recent paper [1], B. M. Stewart presented a structure theorem for the set $S$ of integers $M$, for which $\alpha(M)=\sigma(M)$.

In this paper we will drop the restriction that the divisors need be positive. We will also show that in certain quadratic fields, every integer can be written as a sum of distinct units.

For a given positive integer $M$, let $\beta(M)$ be the number of positive integers $n$ which can be written in the form

$$
n=\sum d
$$

where the $d$ are distinct (positive or negative) divisors of $M$. Let $A$ be the set of all integers $M$ for which $\beta(M)=\sigma(M)$. Let $s(M)$ $=\beta(M) / \sigma(M)$.

2. Structure theorems for $A$. It is clear that if $M=2^{t}, M=3^{t}$ or $M=2^{t} 3^{u}$, then $M$ belongs to $A$.

Lemma 1. If $M$ belongs to $A$ and $p$ is a prime with $(p, M)=1$, then $p^{k} M, k \geqq 1$, belongs to $A$ if and only if $\sigma(M) \geqq(p-1) / 2$.

The condition is necessary in order to represent $(p-1) / 2$ and $(p+1) / 2$ as a sum of distinct divisors of $p^{k} M$.

Conversely, suppose $\sigma(M) \geqq(p-1) / 2$. The proof is by induction. Let $r$ be any integer such that $0 \leqq r \leqq \sigma(M)-1$. Every integer between $r p$ and $r p+\sigma(M)$ can be written as $p \sum_{d / M} d+\sum_{d^{\prime} / M} d^{\prime}$. Every integer from $(r+1) p-\sigma(M)$ to $(r+1) p$ can be written as $p \sum_{d / M} d$ $-\sum_{d^{\prime} / M} d^{\prime}$. It follows from $r p+\sigma(M) \geqq r p+(p+1) / 2=(r+1) p$ $-(p-1) / 2-1 \geqq(r+1) p-\sigma(M)-1$ that every integer up to $p \sigma(M)$ can be written as the desired sum. Furthermore every integer from $p \sigma(M)$ to $p \sigma(M)+\sigma(M)$ can be written as $p \sum_{d / M}+\sum_{d^{\prime} / M}$. Thus $p M$ belongs to $A$.

Since every divisor of $p^{k-1} M$ is also a divisor of $p^{k} M$, the induction hypothesis assures us that every integer from 1 to $\left(p^{k-1} M\right)$ can be

Received by the editors February 9, 1963. 
written as a sum of distinct divisors of $p^{k} M$. Thus every integer from $r p^{k}$ to $r p^{k}+\sigma\left(p^{k-1} M\right)$ can be written as $p^{k} \sum_{d / M} d+\sum_{d^{\prime} / p^{k-1} M} d^{\prime}$ and every integer from $(r+1) p^{k}-\sigma\left(p^{k-1} M\right)$ to $(r+1) p^{k}$ can be written as $p^{k} \sum_{d / M} d-\sum_{d^{\prime} / p^{k-1} M} d^{\prime}$. It follows from $r p^{k}+\sigma\left(p^{k-1} M\right)+1=r p^{k}+1$ $+\sigma(M) \sigma\left(p^{k-1}\right) \geqq r p^{k}+1+(p-1)\left(p^{k}-1\right) / 2(p-1)=r p^{k}+p^{k}$ $-(p-1)\left(p^{k}-1\right) / 2(p-1) \geqq(r+1) p^{k}-\sigma\left(p^{k-1} M\right)$, that every integer up to $p^{k} \sigma(M)$ can be written as the desired sum. Furthermore every integer from $p^{k} \sigma(M)$ to $p^{k} \sigma(M)+\sigma\left(p^{k-1} M\right)$ can be written as $p^{k} \sum_{d / M} d$ $+\sum_{d^{\prime} / p^{k-1} M} d^{\prime}$. Hence $p^{k} M$ belongs to $A$.

Lemma 2. Let $M=\prod_{i=1}^{k} p_{i}^{t_{i}}$, where $p_{i}<p_{j}$ for $i<j$. Let $M_{j}=\prod_{i=1}^{j} p_{i}^{t_{i}}$ for $j \leqq k$ and $p_{0}=M_{0}=1$. If $M$ does not belong to $A$, then $M q^{s}$ does not belong to $A$ for all primes $q>p_{k}$ and all $s \geqq 0$.

Since $M$ does not belong to $A$ there must be a smallest integer $j$ such that $M_{j}$ belongs to $A$ and $M_{j+1}$ does not belong to $A$. By Lemma 1, $\sigma\left(M_{j}\right)<\left(p_{j+1}-1\right) / 2$. Let $R$ be the sum of all divisors of $M$ greater than or equal to $p_{j+1}$. We will show that $R-\left(p_{j+1}-1\right) / 2$ cannot be represented as a sum of distinct divisors of $M$. If $R$ is used positively, the smallest integer which can be represented is $R-\sigma\left(M_{j}\right)>R$ $-\left(p_{j+1}-1\right) / 2$. If any divisor $d \geqq p_{j+1}$ is not used positively, the largest integer which can be represented in the desired manner is $R-d$ $+\sigma\left(M_{j}\right)<R-p_{j+1}+\left(p_{j+1}-1\right) / 2<R-\left(p_{j+1}-1\right) / 2$.

As a direct result of Lemmas 1 and 2, we can now state the following theorem.

TheOREM 1. An integer $M$ belongs to the set $A$ if and only if it has the following factorization as a product of primes:

$$
M=2^{b} 3^{c} \prod_{i=1}^{k} p_{i}^{t_{i}}
$$

where $b$ and $c$ are not both zero, $3<p_{i}<p_{j}$ for $i<j, p_{1} \leqq 2 \sigma\left(2^{b} 3^{c}\right)+1$ and $p_{j+1} \leqq 2 \sigma\left(2^{b} 3^{c} \prod_{i=1}^{j} p_{i}^{t_{i}}\right)+1$ for $1 \leqq j \leqq k-1$.

COROLlary 1. There exist arbitrarily large square free integers in $A$.

Let $M_{k}$ be the product of the first $k$ primes in their natural order. From $p_{j} \leqq M_{j-1}+1 \leqq \sigma\left(M_{j-1}\right)+1<2 \sigma\left(M_{j-1}\right)+1$, it follows that for every $k, M_{k}$ belongs to $A$.

3. Behavior of $s(M)$. It is clear that $0<s(M) \leqq 1$. In order to show that $s(M)$ is everywhere dense on the interval 0 to 1 , we need the following lemma.

Leмma 3. If $p$ is a prime such that $p>2 \sigma(T)+1$, then $\beta(p T)$ $=2 \beta(T)[\beta(T)+1]$. 
Every positive sum of distinct divisors of $p T$ can be written as $n_{1} p+n_{2}$ where $n_{1}$ is zero or any positive integer which can be written as a sum of distinct divisors of $T$, and $n_{2}$ is zero or any positive or negative integer which can be written as a sum of distinct divisors of $T$. The maximum value for $n_{2}$ is $\sigma(T)$. From $n_{1} p+\sigma(T)<n_{1} p+(p-1) / 2$ $<\left(n_{1}+1\right) p-(p-1) / 2<\left(n_{1}+1\right) p-\sigma(T)$, it follows that there is no overlapping. We have $\beta(T)+1$ choices for $n_{1}$ and $2 \beta(T)+1$ choices for $n_{2}$ except when $n_{1}$ is zero. If $n_{1}=0$, then $n_{2}$ must be positive. Thus we have

$$
\beta(p T)=[\beta(T)+1][2 \beta(T)+1)]-[\beta(T)+1]=2 \beta(T)[\beta(T)+1] .
$$

THEOREM 2. $s(M)$ is everywhere dense on the interval 0 to 1.

Given any $x$ and any $y$ such that $0<x<y \leqq 1$, we seek an integer $R$ such that $x<s(R)<y$. Let $R=p M$ where $M$ belongs to $A$ and $p>2 \sigma(M)+1$. By Lemma 3 we have

$$
\begin{aligned}
s(R) & =s(p M)=2 \sigma(M)[\sigma(M)+1] /[(p+1) \sigma(M)] \\
& =2[\sigma(M)+1] /(p+1) .
\end{aligned}
$$

Thus we seek an integer $M$ and a prime $p$ satisfying the above conditions and such that

$$
1 / y<(p+1) / 2[\sigma(M)+1]<1 / x .
$$

Let $u=1 / y, u(1+\epsilon)=1 / x$ and $v=2 u[\sigma(M)+1]$. We know by Theorem 1 that we can find an $M$ belonging to $A$ which is arbitrarily large, so that $v=2 u[\sigma(M)+1]$ can be made arbitrarily large. By the CahenStieltjes theorem we know that for sufficiently large $v$ there exists a prime $p$ such that $v-1<p<(v-1)(1+\epsilon)<v(1+\epsilon)-1$. We now have

$$
\begin{aligned}
v<p+1 & <v(1+\epsilon), \\
2 u[\sigma(M)+1] & <p+1<2 u[\sigma(M)+1](1+\epsilon), \\
1 / \mathrm{y} & <(p+1) / 2[\sigma(M)+1]<1 / x .
\end{aligned}
$$

4. Sums of distinct units in $\operatorname{Ra}(\sqrt{ } 2)$. The integers in the quadratic field $\operatorname{Ra}(\sqrt{ } 2)$ are numbers of the form $x+y \sqrt{ } 2$, where $x$ and $y$ are rational integers and the units are those integers for which $x^{2}-2 y^{2}$ $= \pm 1$. Let $u_{1}=1+\sqrt{ } 2=a_{1}+b_{1} \sqrt{ } 2$ and $u_{j}=u_{1}^{j}=a_{j}+b_{j} \sqrt{ } 2$. It is known that every unit can be written $\pm u_{1}^{n}$ for $n=0, \pm 1, \pm 2, \cdots$. The units may now be divided into mutually exclusive sets, $V_{0}=(+1,-1)$, $V_{j}=\left(u_{j},-u_{j}, 1 / u_{j},-1 / u_{j}\right)$ for $j=1,2, \cdots$. Let $B_{k}=\sum_{i=1}^{k} b_{i}$. We will make use of the following relationships:

1. $b_{k+1}=a_{k}+b_{k}<a_{k}+2 b_{k}=a_{k+1}$ for $k \geqq 1$,

2. $2 B_{k-1} \leqq a_{k}+b_{k} \leqq 4 B_{k-1}$ for $k \geqq 3$. 
We will represent any integer $(P=x+y \sqrt{ } 2)$ in $\operatorname{Ra}(\sqrt{ } 2)$ as a lattice point $(P)$ in the plane, whose rectangular coordinates are $(x, y)$. We will fill the plane with concentric squares whose diagonals are the coordinate axes. The length of the diagonal of the $k$ square is $4 B_{k}$. It will be shown that every integer lying in the $k$ square can be represented as a sum of distinct units belonging to the sets $V_{0}, V_{1}, \cdots, V_{k}$.

TheOREM 3. Every integer in $\mathrm{Ra}(\sqrt{ } 2)$ can be written as a sum of distinct units.

By inspection it can be seen that every integer in the first three squares can be represented as a sum of distinct units in the sets $V_{0}, V_{1}$, $V_{2}$ and $V_{3}$. Let us assume that every integer in the $k-1$ square can be written as a sum of distinct units in the sets $V_{0}, V_{1}, \cdots, V_{k-1}$ and prove the theorem by induction.

Consider any point inside the $k$ square. Since the square and all sets of units are symmetric with respect to both axes, there is no loss in generality by assuming that the point lies in the first quadrant, on the positive $x$ axis or on the positive $y$ axis. If the point lies inside the $k-1$ square, a required representation is assured by the induction hypothesis. Thus we have:

$$
2 B_{k-1}<x+y \leqq 2 B_{k}, \quad y \geqq 0, \quad x \geqq 0 .
$$

We divide the possibilities into three cases. In Case 1 the point lies above the line $y-x=2\left(b_{k}-B_{k-1}\right)$. In Case 2 the point lies below the line $y-x=2\left(B_{k-1}-a_{k}\right)$. In Case 3 the point lies on or between these two lines.

Case 1. $2 B_{k-1}<x+y \leqq 2 B_{k}$,

$$
y-x>2\left(b_{k}-B_{k-1}\right), \quad y \geqq 0, \quad x \geqq 0 .
$$

If we subtract $2 b_{k}$ from $y$, we obtain a point $P^{\prime}$ with rectangular coordinates $x^{\prime}=x$ and $y^{\prime}=y-2 b_{k}$.

$$
\begin{aligned}
& x^{\prime}+y^{\prime} \leqq 2 B_{k}-2 b_{k}=2 B_{k-1}, \\
& y^{\prime}-x^{\prime}>2\left(b_{k}-B_{k-1}\right)-2 b_{k}=-2 B_{k-1}, \text { and } x^{\prime} \geqq 0 .
\end{aligned}
$$

Thus the point $P^{\prime}$ lies inside the $k-1$ square and by the induction hypothesis can be represented as a sum $S_{k-1}$ of distinct units in the first $k$ sets of units. $P=S_{k-1}+2 b_{k} \sqrt{ } 2=S_{k-1}+\left(a_{k}+b_{k} \sqrt{ } 2\right)$ $+\left(-a_{k}+b_{k} \sqrt{ } 2\right)$ and the theorem is proved in Case 1.

Case 2. $2 B_{k-1}<x+y \leqq 2 B_{k}$,

$$
y-x<2\left(B_{k-1}-a_{k}\right), \quad y \geqq 0, \quad x \geqq 0 .
$$


If we subtract $2 a_{k}$ from $x$, we obtain a point $P^{\prime}$ with coordinates $x^{\prime}=x-2 a_{k}$ and $y^{\prime}=y$.

$$
\begin{aligned}
& x^{\prime}+y^{\prime} \leqq 2 B_{k}-2 a_{k} \leqq 2 B_{k}-2 b_{k}=2 B_{k-1}, \\
& y^{\prime}-x^{\prime}<2\left(B_{k-1}-a_{k}\right)+2 a_{k}=2 B_{k-1}, \quad y^{\prime} \geqq 0 .
\end{aligned}
$$

Thus the point $P^{\prime}$ lies inside the $k-1$ square and by the induction hypothesis can be represented as a sum $S_{k-1}$ of distinct units belonging to the first $k$ sets of units. $P=S_{k-1}+2 a_{k}=S_{k-1}+\left(a_{k}+b_{k} \sqrt{ } 2\right)$ $+\left(a_{k}-b_{k} \sqrt{ } 2\right)$. Thus the theorem is proved for Case 2 .

Case 3. $2 B_{k-1}<x+y \leqq 2 B_{k}$,

$$
2\left(b_{k}-B_{k-1}\right) \geqq y-x \geqq 2\left(B_{k-1}-a_{k}\right) .
$$

If we subtract $a_{k}$ from $x$ and $b_{k}$ from $y$, we obtain a point $P^{\prime}$ with coordinates $x^{\prime}=x-a_{k}$ and $y^{\prime}=y-b_{k}$. Applying (2) we obtain the following inequalities:

$$
\begin{aligned}
& x^{\prime}+y^{\prime}>2 B_{k-1}-a_{k}-b_{k} \geqq 2 B_{k-1}-4 B_{k-1}=-2 B_{k-1}, \\
& x^{\prime}+y^{\prime} \leqq 2 B_{k}-a_{k}-b_{k}<2 B_{k}-2 b_{k}=2 B_{k-1}, \\
& y^{\prime}-x^{\prime} \geqq 2 B_{k-1}-2 a_{k}-b_{k}+a_{k}=2 B_{k-1}-a_{k}-b_{k} \geqq-2 B_{k-1}, \\
& y^{\prime}-x^{\prime} \leqq 2 b_{k}-2 B_{k-1}-b_{k}+a_{k}=a_{k}+b_{k}-2 B_{k-1} \leqq 2 B_{k-1} .
\end{aligned}
$$

Thus the point $P^{\prime}$ lies inside the $k-1$ square and by the induction hypothesis can be represented as a sum $S_{k-1}$ of distinct units in the first $k$ sets of units $V_{0}, V_{1}, \cdots, V_{k-1} . P=S_{k-1}+\left(a_{k}+b_{k} \sqrt{ } 2\right)$ and the theorem is proved.

5. Sums of distinct units in $\operatorname{Ra}(\sqrt{ } m)$. Theorem 3 is true for $m=5$. A method of proof identical to the method used in Theorem 3 may be used to verify this. Theorem 3 is not true for $m=3,6$, or 7 . Clearly the theorem is not true for $m<0$. It is conjectured by the author that Theorem 3 is true only for $m=2$ and $m=5$.

\section{REFERENCE}

1. B. M. Stewart, Sums of distinct divisors, Amer. J. Math. 76 (1954), 779-785.

Frankin and Marshall College 